\title{
Statistical „Pole Position” Leadership between Lamborghini versus Ferrari
}

\author{
Gabriela OPAIT ${ }^{\star}$
}

\begin{tabular}{l}
\hline \multicolumn{1}{c}{ A R T I C L E I N F O } \\
\hline Article history: \\
Accepted January 2019 \\
Available online April 2019 \\
\hline JEL Classification \\
C1, C12, C2 \\
Keywords: \\
Exotic car, Hybrid electric car, \\
Style kinetic energy recovery \\
system \\
\hline
\end{tabular}

A B S T R A C T

Lamborghini and Ferrari represents two great hyper powers in the worldwide sphere of the firms which product cars of high performance. In each year, the both firms highlight new models cars at the great salons of expositions, where the prototypes innovated fascinate through shapes, horsepower, engine systems, top speed, electrical performances and more indexes which reflect the „implant”concerning the management of the quality.

(C) 2019 EAI. All rights reserved.

\section{Introduction}

This statistical highlight puts in evidence the competition between two great firms which conquered the lovers of performant cars, namely Lamborghini and Ferrari. The target of this research follows the achievement of the forecasts concerning the values of the levels touched in the sales of vehicles by the both firms, Lamborghini and Ferrari, for to reflect the real leadership highlighted in „pole positions”. The first side of this statistical research brings in first plane, how we can to select the trend concerning the number of Lamborghini vehicles selled between 2006-2018, at the worldwide level. The second side guides the readers in the achievement of the methodology regarding the selection of the model which reflects the volum of Ferrari vehicles merchandised in all over the world, in the same interval 2006-2018. The methods used are the „Regression Method” and the „Variation Method” for to achieve the forecasts regarding the values of Lamborghini and Ferrari vehicles selled at the worldwide level. In 1823, Johann Carl Friedrich Gauss drawed up the „Least Squares Method", where the target is the identification of the parameters which belong to the model puts in the sphere of the research.

2. The design of the projection concerning the volum of the worldwide sales for the Lamborghini cars, between 2006-2018

Table 1 The values which which have as essence the „fizzy" architectures of the both champions Lamborghini\&Ferrari concerning the series of successes in the worldwide sales for vehicles

\begin{tabular}{|c|c|c|}
\hline YEARS & $\begin{array}{c}\text { LAMBORGHINI } \\
\text { WORLDWIDE NUMBER OF VEHICLES }\end{array}$ & $\begin{array}{c}\text { FERRARI } \\
\text { WORLDWIDE NUMBER OF VEHICLES }\end{array}$ \\
\hline 2006 & 2087 & 5671 \\
\hline 2007 & 2406 & 6465 \\
\hline 2008 & 2430 & 6587 \\
\hline 2009 & 1515 & 6250 \\
\hline 2010 & 1302 & 6461 \\
\hline 2011 & 1602 & 7001 \\
\hline 2012 & 2083 & 7318 \\
\hline 2013 & 2121 & 6922 \\
\hline 2014 & 2530 & 7255 \\
\hline 2015 & 3245 & 7664 \\
\hline 2016 & 3457 & 8014 \\
\hline 2017 & 3815 & 8398 \\
\hline 2018 & 5750 & 9251 \\
\hline
\end{tabular}

^Dunarea de Jos University of Galati, Romania. E-mail address: gabriela.opait@ugal.ro 
- if the „sophisticated design” for $\xi$ variable, namely the Lamborghini worldwide sales, is this representation $\xi_{t_{i}}=a+b \cdot t_{i}, a$ and $b$ will be [3]:

$$
\begin{aligned}
& S=\sum_{i=1}^{n}\left(\xi_{i}-\xi_{t i}\right)^{2}=\min \Leftrightarrow S=\sum_{i=1}^{n}\left(\xi_{i}-a-b t_{i}\right)^{2}=\min \\
& \left\{\begin{array} { l } 
{ \frac { \partial S } { \partial a } = 0 } \\
{ \frac { \partial S } { \partial b } = 0 }
\end{array} \Rightarrow \left\{\begin{array} { l } 
{ 2 \sum _ { 1 = 1 } ^ { n } ( \xi _ { i } - a - b t _ { i } ) ( - 1 ) = 0 / ( - \frac { 1 } { 2 } ) } \\
{ 2 \sum _ { 1 = 1 } ^ { n } ( \xi _ { i } - a - b t _ { i } ) ( - t _ { i } ) = 0 / ( - \frac { 1 } { 2 } ) }
\end{array} \Rightarrow \left\{\begin{array}{l}
n a+b \sum_{i=1}^{n} t_{i}=\sum_{i=1}^{n} \xi_{i} \\
a \sum_{i=1}^{n} t_{i}+b \sum_{i=1}^{n} t_{i}^{2}=\sum_{i=1}^{n} \xi_{i} t_{i}
\end{array} \Rightarrow\right.\right.\right.
\end{aligned}
$$

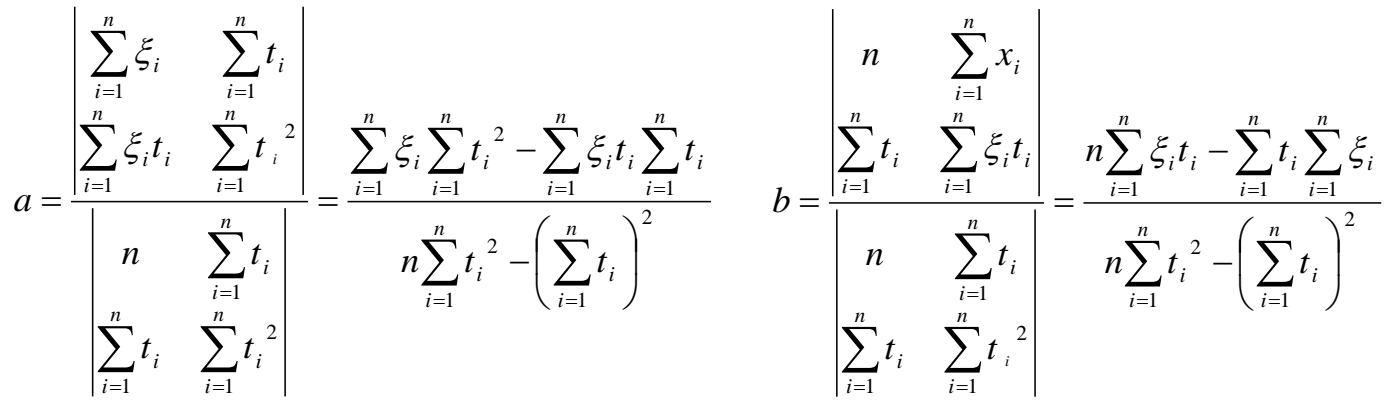

Table 2 The „conglomerate” of the values which joins under the incidence of the reflection

\begin{tabular}{|c|c|c|c|c|c|c|}
\hline \multirow{2}{*}{ YEARS } & \multirow{2}{*}{$\begin{array}{l}\text { LAMBORGHINI } \\
\text { WORLDWIDE } \\
\text { NUMBER OF } \\
\text { VEHICLES } \\
\left(\xi_{i}\right)\end{array}$} & \multicolumn{5}{|c|}{ LINEAR TENDENCY } \\
\hline & & $t_{i}$ & $t_{i}^{2}$ & $t_{i} \xi_{i}$ & $\xi_{t_{i}}=a+b t_{i}$ & $\left|\xi_{i}-\xi_{t_{i}}\right|$ \\
\hline 2006 & 2087 & -6 & 36 & -12522 & 1280,362638 & 807 \\
\hline 2007 & 2406 & -5 & 25 & -12030 & 1507,263737 & 899 \\
\hline 2008 & 2430 & -4 & 16 & -9720 & 1734,164835 & 696 \\
\hline 2009 & 1515 & -3 & 9 & -4545 & 1961,065934 & 446 \\
\hline 2010 & 1302 & -2 & 4 & -2604 & 2187,967033 & 886 \\
\hline 2011 & 1602 & -1 & 1 & -1602 & 2414,868132 & 813 \\
\hline 2012 & 2083 & 0 & 0 & 0 & 2641,769231 & 559 \\
\hline 2013 & 2121 & 1 & 1 & 2121 & 2868,670330 & 748 \\
\hline 2014 & 2530 & 2 & 4 & 5060 & 3095,571429 & 566 \\
\hline 2015 & 3245 & 3 & 9 & 9735 & 3322,472528 & 77 \\
\hline 2016 & 3457 & 4 & 16 & 13828 & 3549,373627 & 92 \\
\hline 2017 & 3815 & 5 & 25 & 19075 & 3776,274726 & 39 \\
\hline 2018 & 5750 & 6 & 36 & 34500 & 4003,175824 & 1747 \\
\hline TOTAL & 34343 & & 182 & 41296 & 34343 & 8375 \\
\hline
\end{tabular}
concerning the linear pattern for the volum of Lamborghini cars selled at worldwide level

$$
\begin{aligned}
& a=\frac{34343 \cdot 182-41296 \cdot 0}{13 \cdot 182-0^{2}}=2641,769231 \\
& b=\frac{13 \cdot 41296-34343 \cdot 0}{13 \cdot 182-0^{2}}=226,9010989
\end{aligned}
$$




$$
v_{I}=\left[\frac{\sum_{i=-m}^{m}\left|\xi_{i}-\xi_{t_{i}}^{I}\right|}{n}: \frac{\sum_{i=-m}^{m} x_{i}}{n}\right] \cdot 100=\frac{\sum_{i=-m}^{m}\left|\xi_{i}-\xi_{t_{i}}^{I}\right|}{\sum_{i=-m}^{m} \xi_{i}} \cdot 100=\frac{8375}{34343} \cdot 100=24,386 \%
$$

- if the „sophisticated design” for $\xi$ variable, namely the Lamborghini worldwide sales, is this representation $\xi_{t_{i}}=a+b \cdot t_{i}+c t_{i}^{2}, a$ and $b$ will be [3]:

$$
\begin{aligned}
& S=\sum_{i=1}^{n}\left(\xi_{i}-x_{t i}\right)^{2}=\min \Leftrightarrow S=\sum_{i=1}^{n}\left(\xi_{i}-a-b t_{i}-c t_{i}^{2}\right)^{2}=\min \\
& \left\{\begin{array} { l } 
{ \frac { \partial S } { \partial a } = 0 } \\
{ \frac { \partial S } { \partial b } = 0 } \\
{ \frac { \partial S } { \partial c } = 0 }
\end{array} \left\{\begin{array}{l}
2 \sum_{1=1}^{n}\left(\xi_{i}-a-b t_{i}-c t_{i}^{2}\right)(-1)=0 /\left(-\frac{1}{2}\right) \\
2 \sum_{1=1}^{n}\left(\xi_{i}-a-b t_{i}-c t_{i}^{2}\right)\left(-t_{i}\right)=0 /\left(-\frac{1}{2}\right) \\
2 \sum_{i=1}^{n}\left(\xi_{i}-a-b t_{i}-c t_{i}^{2}\right)\left(-t_{i}^{2}\right)=0 /\left(-\frac{1}{2}\right)
\end{array} \Rightarrow\right.\right. \\
& \left\{\begin{array}{l}
n \cdot a+b \sum_{i=1}^{n} t_{i}+c \sum_{i=1}^{n} t_{i}^{2}=\sum_{i=1}^{n} \xi_{i} \\
a \sum_{i=1}^{n} t_{i}+b \cdot \sum_{i=1}^{n} t_{i}^{2}+c \sum_{i=1}^{n} t_{i}^{3}=\sum_{i=1}^{n} t_{i} \cdot \xi_{i}
\end{array}\right. \\
& a \cdot \sum_{i=1}^{n} t_{i}^{2}+b \sum_{i=1}^{n} t_{i}^{3}+c \sum_{i=1}^{n} t_{i}^{4}=\sum_{i=1}^{n} t_{i}^{2} \cdot \xi_{i} \\
& a=\frac{\sum_{i=1}^{n} t_{i}^{4} \sum_{i=1}^{n} \xi_{i}-\sum_{i=1}^{n} t_{i}^{2} \sum_{i=1}^{n} t_{i}^{2} \cdot \xi_{i}}{n \sum_{i=1}^{n} t_{i}^{4}-\left(\sum_{i=1}^{n} t_{i}^{2}\right)^{2}} ; \quad b=\frac{\sum_{i=1}^{n} \xi_{i} t_{i}}{\sum_{i=1}^{n} t_{i}^{2}} ; c=\frac{n \cdot \sum_{i=1}^{n} t_{i}^{2} \cdot \xi_{i}-\sum_{i=1}^{n} t_{i}^{2} \cdot \sum_{i=1}^{n} \xi_{i}}{n \sum_{i=1}^{n} t_{i}^{4}-\left(\sum_{i=1}^{n} t_{i}^{2}\right)^{2}}
\end{aligned}
$$

\begin{tabular}{|c|c|c|c|c|c|c|c|}
\hline \multirow[b]{2}{*}{ YEARS } & \multirow{2}{*}{$\begin{array}{c}\text { LAMBORGHINI } \\
\text { WORLDWIDE } \\
\text { NUMBER OF VEHICLES } \\
\left(\xi_{i}\right)\end{array}$} & \multicolumn{6}{|c|}{ PARABOLIC TENDENCY } \\
\hline & & $t_{i}$ & $t_{i}^{2}$ & $t_{i}^{4}$ & $t_{i}^{2} \xi_{i}$ & $\xi_{t_{i}}=a+b t_{i}+c t_{i}^{2}$ & $\left|\xi_{i}-\xi_{t_{i}}\right|$ \\
\hline 2006 & 2087 & -6 & 36 & 1296 & 75132 & 2521,43956 & 434 \\
\hline 2007 & 2406 & -5 & 25 & 625 & 60150 & 2127,802198 & 278 \\
\hline 2008 & 2430 & -4 & 16 & 256 & 38880 & 1846,990010 & 583 \\
\hline 2009 & 1515 & -3 & 9 & 81 & 13635 & 1679,002997 & 164 \\
\hline 2010 & 1302 & -2 & 4 & 16 & 5208 & 1623,841159 & 322 \\
\hline 2011 & 1602 & -1 & 1 & 1 & 1602 & 1681,504496 & 79 \\
\hline 2012 & 2083 & 0 & 0 & 0 & 0 & 1851,993007 & 231 \\
\hline 2013 & 2121 & 1 & 1 & 1 & 2121 & 2135,306693 & 14 \\
\hline 2014 & 2530 & 2 & 4 & 16 & 10120 & 2531,445554 & 1 \\
\hline 2015 & 3245 & 3 & 9 & 81 & 29205 & 3040,409590 & 205 \\
\hline 2016 & 3457 & 4 & 16 & 256 & 55312 & 3662,198801 & 205 \\
\hline 2017 & 3815 & 5 & 25 & 625 & 95375 & 4396,813187 & 582 \\
\hline 2018 & 5750 & 6 & 36 & 1296 & 207000 & 5244,252747 & 506 \\
\hline TOTAL & 34343 & & 182 & 4550 & 593740 & 34343 & 3604 \\
\hline
\end{tabular}

Table 3 The „conglomerate” of the values which joins under the incidence of the reflection concerning the quadratic pattern for the volum of Lamborghini cars selled at worldwide level 


$$
\begin{gathered}
a=\frac{\sum_{i=1}^{n} t_{i}^{4} \sum_{i=1}^{n} \xi_{i}-\sum_{i=1}^{n} t_{i}^{2} \sum_{i=1}^{n} t_{i}^{2} \cdot \xi_{i}}{n \sum_{i=1}^{n} t_{i}^{4}-\left(\sum_{i=1}^{n} t_{i}^{2}\right)^{2}}=\frac{4550 \cdot 34343-182 \cdot 593740}{13 \cdot 4550-182^{2}}=1851,993007 \\
b=\frac{\sum_{i=1}^{n} \xi_{i} t_{i}}{\sum_{i=1}^{n} t_{i}^{2}}=\frac{41296}{182}=226,9010989 \\
c=\frac{n \cdot \sum_{i=1}^{n} t_{i}^{2} \cdot \xi_{i}-\sum_{i=1}^{n} t_{i}^{2} \cdot \sum_{i=1}^{n} \xi_{i}}{n \sum_{i=1}^{n} t_{i}^{4}-\left(\sum_{i=1}^{n} t_{i}^{2}\right)^{2}}=\frac{13 \cdot 593740-182 \cdot 34343}{13 \cdot 4550-182^{2}}=56,41258741 \\
v_{I I}=\left[\frac{\sum_{i=-m}^{m}\left|\xi_{i}-\xi_{t_{i}}^{I I}\right|}{n}: \frac{\sum_{i=-m}^{m} x_{i}}{n}\right] \cdot 100=\frac{\sum_{i=-m}^{m}\left|\xi_{i}-\xi_{t_{i}}^{I I}\right|}{\sum_{i=-m}^{m} \xi_{i}} \cdot 100=\frac{3604}{34343} \cdot 100=10,49 \%
\end{gathered}
$$

- if the „sophisticated design” for $\xi$ variable, namely the Lamborghini worlwide sales, is this representation $\xi_{t_{i}}=a b^{t_{i}}, a$ and $b$ will be [3]:

$$
\begin{aligned}
& S=\sum_{i=1}^{n}\left(\lg \xi_{i}-\lg \xi_{t_{i}}\right)^{2}=\min \Leftrightarrow S=\sum_{i=1}^{n}\left(\lg \xi_{i}-\lg a-t_{i} \lg b\right)^{2}=\min \\
& \left\{\begin{array} { l } 
{ \frac { \partial S } { \partial \operatorname { l g } a } = 0 } \\
{ \frac { \partial S } { \partial \operatorname { l g } b } = 0 }
\end{array} \Rightarrow \left\{\begin{array}{l}
2 \sum_{1=1}^{n}\left(\lg \xi_{i}-\lg a-t_{i} \lg b\right)(-1)=0 /\left(-\frac{1}{2}\right) \\
2 \sum_{1=1}^{n}\left(\lg \xi_{i}-\lg a-t_{i} \lg b\right)\left(-t_{i}\right)=0 /\left(-\frac{1}{2}\right)
\end{array} \Rightarrow\right.\right. \\
& \left\{\begin{array}{l}
n \cdot \lg a+\lg b \cdot \sum_{i=1}^{n} t_{i}=\sum_{i=1}^{n} \lg \xi_{i} \\
\lg a \sum_{i=1}^{n} t_{i}+\lg b \cdot \sum_{i=1}^{n} t_{i}^{2}=\sum_{i=1}^{n} t_{i} \cdot \lg \xi_{i}
\end{array}\right. \\
& \lg a=\frac{\left|\begin{array}{cc}
\sum_{i=1}^{n} \lg \xi_{i} & \sum_{i=1}^{n} t_{i} \\
\sum_{i=1}^{n} t_{i} \lg \xi_{i} & \sum_{i=1}^{n} t_{i}{ }^{2}
\end{array}\right|}{\left|\begin{array}{cc}
n & \sum_{i=1}^{n} t_{i} \\
\sum_{i=1}^{n} t_{i} & \sum_{i=1}^{n} t_{i}{ }^{2}
\end{array}\right|}=\frac{\sum_{i=1}^{n} \lg \xi_{i} \sum_{i=1}^{n} t_{i}{ }^{2}-\sum_{i=1}^{n} t_{i} \lg \xi_{i} \sum_{i=1}^{n} t_{i}}{n \sum_{i=1}^{n} t_{i}{ }^{2}-\left(\sum_{i=1}^{n} t_{i}\right)^{2}}
\end{aligned}
$$




$$
\lg b=\frac{\left|\begin{array}{cc}
n & \sum_{i=1}^{n} \lg \xi_{i} \\
\sum_{i=1}^{n} t_{i} & \sum_{i=1}^{n} t_{i} \lg \xi_{i}
\end{array}\right|}{\left|\begin{array}{cc}
n & \sum_{i=1}^{n} t_{i} \\
\sum_{i=1}^{n} t_{i} & \sum_{i=1}^{n} t_{i}^{2}
\end{array}\right|}=\frac{n \cdot \sum_{i=1}^{n} t_{i} \lg \xi_{i}-\sum_{i=1}^{n} \lg \xi_{i} \sum_{i=1}^{n} t_{i}}{n \sum_{i=1}^{n} t_{i}{ }^{2}-\left(\sum_{i=1}^{n} t_{i}\right)^{2}}
$$

Table 4 The "conglomerate” of the values which joins under the incidence of the reflection concerning the exponential pattern for the volum of Lamborghini cars selled at worldwide level

\begin{tabular}{|c|c|c|c|c|c|c|}
\hline \multirow{2}{*}{ YEARS } & \multirow{2}{*}{$\begin{array}{l}\text { LAMBORGHINI } \\
\text { WORLDWIDE } \\
\text { NUMBER OF } \\
\text { VEHICLES } \\
\left(\xi_{i}\right)\end{array}$} & \multicolumn{5}{|c|}{ EXPONENTIAL TENDENCY } \\
\hline & & $\lg \xi_{i}$ & $t_{i} \lg \xi_{i}$ & $\begin{array}{c}\lg \xi_{t_{i}}= \\
=\lg a+t_{i} \lg b\end{array}$ & $\xi_{t_{i}}=a b^{t_{i}}$ & $\left|\xi_{i}-\xi_{t_{i}}\right|$ \\
\hline 2006 & 2087 & 3,319522449 & $-19,91713469$ & 3,190572898 & 1550,861083 & 536 \\
\hline 2007 & 2406 & 3,381295623 & $-16,90647812$ & 3,223240715 & 1672,017101 & 734 \\
\hline 2008 & 2430 & 3,385606274 & $-13,54242509$ & 3,255908532 & 1802,638042 & 627 \\
\hline 2009 & 1515 & 3,180412633 & $-9,541237899$ & 3,288576349 & 1943,463323 & 428 \\
\hline 2010 & 1302 & 3,114610984 & $-6,229221968$ & 3,321244166 & 2095,290124 & 793 \\
\hline 2011 & 1602 & 3,204662512 & $-3,204662512$ & 3,353911983 & 2258,977904 & 657 \\
\hline 2012 & 2083 & 3,318689270 & 0 & 3,386579800 & 2435,453264 & 352 \\
\hline 2013 & 2121 & 3,326540669 & 3,326540669 & 3,419247617 & 2625,715192 & 505 \\
\hline 2014 & 2530 & 3,403120521 & 6,806241042 & 3,451915434 & 2830,84072 & 301 \\
\hline 2015 & 3245 & 3,511214701 & 10,5336441 & 3,484583251 & 3051,991017 & 193 \\
\hline 2016 & 3457 & 3,538699380 & 14,15479752 & 3,517251068 & 3290,417968 & 167 \\
\hline 2017 & 3815 & 3,581494542 & 17,90747271 & 3,549918885 & 3547,471254 & 268 \\
\hline 2018 & 5750 & 3,759667845 & 22,55800707 & 3,582586702 & 3824,606000 & 1926 \\
\hline TOTAL & 34343 & 44,0255374 & 5,945542830 & & & 7487 \\
\hline
\end{tabular}

$$
\begin{gathered}
\lg a=\frac{44,0255374 \cdot 182-5,94554283 \cdot 0}{13 \cdot 182-0^{2}}=3,3865798 \\
\lg b=\frac{13 \cdot 5,94554283-44,0255374 \cdot 0}{13 \cdot 182-0^{2}}=0,032667817 \\
v_{\exp }=\left[\frac{\sum_{i=-m}^{m}\left|\xi_{i}-\xi_{t_{i}}^{\exp }\right|}{n}: \frac{\sum_{i=-m}^{m} x_{i}}{n}\right] \cdot 100=\frac{\sum_{i=-m}^{m}\left|\xi_{i}-\xi_{t_{i}}^{\exp }\right|}{\sum_{i=-m}^{m} \xi_{i}} \cdot 100=\frac{7487}{34343} \cdot 100=21,81 \% \\
v_{I I}=10,49 \%<v_{\exp }=21,81 \%<v_{I}=24,386 \%
\end{gathered}
$$

The "design of the rally" regarding the projection which belongs to the Lamborghini worldwide sales, reveals a quadratic pattern $\xi_{t_{i}}=a+b \cdot t_{i}+c t_{i}^{2}$ 


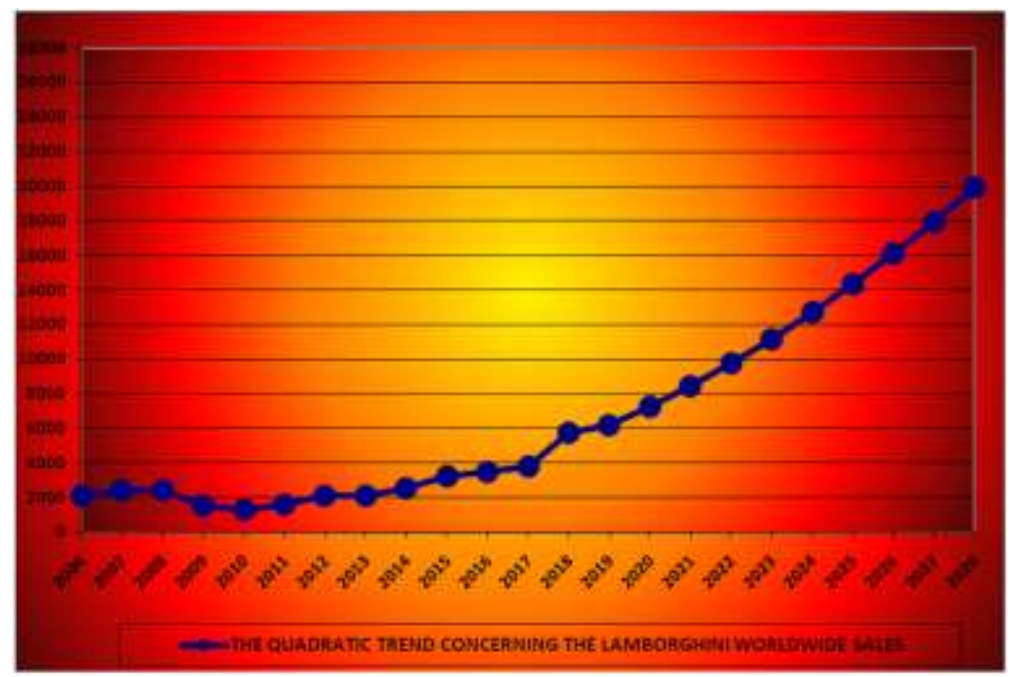

Graph 1 The design of the quadratic pattern concerning the worldwide sales for the Lamborghini cars

. The design of the projection regarding the worldwide sales for the Ferrari cars, between 2006-2018

- if the „sophisticated design” for $\omega$ characteristic, namely the Ferrari worldwide sales, is this mirroring $\omega_{t_{i}}=a+b \cdot t_{i}, a$ and $b$ will be [3]:

$$
\begin{aligned}
& S=\sum_{i=1}^{n}\left(\omega_{i}-\omega_{t i}\right)^{2}=\min \Leftrightarrow S=\sum_{i=1}^{n}\left(\omega_{i}-a-b t_{i}\right)^{2}=\min \\
& \left\{\begin{array} { l } 
{ \frac { \partial S } { \partial a } = 0 } \\
{ \frac { \partial S } { \partial b } = 0 }
\end{array} \Rightarrow \left\{\begin{array} { l } 
{ 2 \sum _ { 1 = 1 } ^ { n } ( \omega _ { i } - a - b t _ { i } ) ( - 1 ) = 0 / ( - \frac { 1 } { 2 } ) } \\
{ 2 \sum _ { 1 = 1 } ^ { n } ( \omega _ { i } - a - b t _ { i } ) ( - t _ { i } ) = 0 / ( - \frac { 1 } { 2 } ) }
\end{array} \Rightarrow \left\{\begin{array}{l}
n a+b \sum_{i=1}^{n} t_{i}=\sum_{i=1}^{n} \omega_{i} \\
a \sum_{i=1}^{n} t_{i}+b \sum_{i=1}^{n} t_{i}^{2}=\sum_{i=1}^{n} \omega_{i} t_{i}
\end{array} \Rightarrow\right.\right.\right. \\
& a=\frac{\left|\begin{array}{ll}
\sum_{i=1}^{n} \omega_{i} & \sum_{i=1}^{n} t_{i} \\
\sum_{i=1}^{n} \omega_{i} t_{i} & \sum_{i=1}^{n} t_{i}{ }^{2}
\end{array}\right|}{\left|\begin{array}{ll}
n & \sum_{i=1}^{n} t_{i} \\
\sum_{i=1}^{n} t_{i} & \sum_{i=1}^{n} t_{i}{ }^{2}
\end{array}\right|}=\frac{\sum_{i=1}^{n} \omega_{i} \sum_{i=1}^{n} t_{i}{ }^{2}-\sum_{i=1}^{n} \omega_{i} t_{i} \sum_{i=1}^{n} t_{i}}{n \sum_{i=1}^{n} t_{i}{ }^{2}-\left(\sum_{i=1}^{n} t_{i}\right)^{2}} \quad b=\frac{\left|\begin{array}{cc}
n & \sum_{i=1}^{n} \omega_{i} \\
\sum_{i=1}^{n} t_{i} & \sum_{i=1}^{n} \omega_{i} t_{i}
\end{array}\right|}{\mid n} \sum_{i=1}^{n} t_{i}^{n} \sum_{i=1}^{n} \omega_{i} t_{i}-\sum_{i=1}^{n} t_{i} \sum_{i=1}^{n} \omega_{i}
\end{aligned}
$$

\begin{tabular}{|c|c|c|c|c|c|c|}
\hline \multirow{2}{*}{ YEARS } & \multirow{2}{*}{$\begin{array}{c}\text { FERRARI } \\
\text { WORLDWIDE } \\
\text { NUMBER OF } \\
\text { VEHICLES } \\
\left(\omega_{i}\right)\end{array}$} & \multicolumn{5}{|c|}{ LINEAR TENDENCY } \\
\hline & & $t_{i}$ & $t_{i}^{2}$ & $t_{i} \omega_{i}$ & $\omega_{t_{i}}=a+b t_{i}$ & $\left|\omega_{i}-\omega_{t_{i}}\right|$ \\
\hline 2006 & 5671 & -6 & 36 & -34026 & 5769,195978 & 98 \\
\hline 2007 & 6465 & -5 & 25 & -32325 & 6003,12088 & 462 \\
\hline 2008 & 6587 & -4 & 16 & -26348 & 6237,219781 & 350 \\
\hline
\end{tabular}

Table 5 The „conglomerate” of the statistical data which joins under the incidence of the reflection concerning the linear pattern for the volum of Ferrari cars selled at worldwide level 


\begin{tabular}{|c|c|c|c|c|c|c|}
\hline \multirow{2}{*}{ YEARS } & \multirow{2}{*}{$\begin{array}{c}\text { FERRARI } \\
\text { WORLDWIDE } \\
\text { NUMBER OF } \\
\text { VEHICLES } \\
\left(\omega_{i}\right)\end{array}$} & \multicolumn{5}{|c|}{ LINEAR TENDENCY } \\
\hline & & $t_{i}$ & $t_{i}^{2}$ & $t_{i} \omega_{i}$ & $\omega_{t_{i}}=a+b t_{i}$ & $\omega_{i}-\omega_{t_{i}}$ \\
\hline 2009 & 6250 & -3 & 9 & -18750 & 6471,318682 & 221 \\
\hline 2010 & 6461 & -2 & 4 & -12922 & 6705,417583 & 244 \\
\hline 2011 & 7001 & -1 & 1 & -7001 & 6939,516484 & 61 \\
\hline 2012 & 7318 & 0 & 0 & 0 & 7173,615385 & 144 \\
\hline 2013 & 6922 & 1 & 1 & 6922 & 7407,714286 & 486 \\
\hline 2014 & 7255 & 2 & 4 & 14510 & 7641,813187 & 387 \\
\hline 2015 & 7664 & 3 & 9 & 22992 & 7875,912088 & 212 \\
\hline 2016 & 8014 & 4 & 16 & 32056 & 8110,010989 & 96 \\
\hline 2017 & 8398 & 5 & 25 & 41990 & 8344,109891 & 54 \\
\hline 2018 & 9251 & 6 & 36 & 55506 & 8578,208792 & 673 \\
\hline TOTAL & 93257 & & 182 & 42606 & 93257 & 3488 \\
\hline
\end{tabular}

$$
\begin{gathered}
a=\frac{93257 \cdot 182-42606 \cdot 0}{13 \cdot 182-0^{2}}=7173,615385 ; b=\frac{42606}{182}=234,0989011 \\
v_{I}=\left[\frac{\sum_{i=-m}^{m}\left|\omega_{i}-y_{t_{i}}^{I}\right|}{n}: \frac{\sum_{i=-m}^{m} \omega_{i}}{n}\right] \cdot 100=\frac{\sum_{i=-m}^{m}\left|\omega_{i}-\omega_{t_{i}}^{I}\right|}{\sum_{i=-m}^{m} \omega_{i}} \cdot 100=\frac{3488}{93257} \cdot 100=3,74 \%
\end{gathered}
$$

- if the "sophisticated design” for $\omega$ characteristic, namely the Ferrari worldwide sales, is this mirroring $\omega_{t_{i}}=a+b \cdot t_{i}+c t_{i}^{2}, a$ and $b$ will be [3]:

$$
\begin{gathered}
\left\{\begin{array}{l}
n \cdot a+b \sum_{i=1}^{n} t_{i}+c \sum_{i=1}^{n} t_{i}^{2}=\sum_{i=1}^{n} \omega_{i} \\
a \sum_{i=1}^{n} t_{i}+b \cdot \sum_{i=1}^{n} t_{i}^{2}+c \sum_{i=1}^{n} t_{i}^{3}=\sum_{i=1}^{n} t_{i} \cdot \omega_{i} \\
a \cdot \sum_{i=1}^{n} t_{i}^{2}+b \sum_{i=1}^{n} t_{i}^{3}+c \sum_{i=1}^{n} t_{i}^{4}=\sum_{i=1}^{n} t_{i}^{2} \cdot \omega_{i}
\end{array}\right. \\
a=\frac{\sum_{i=1}^{n} t_{i}^{4} \sum_{i=1}^{n} \omega_{i}-\sum_{i=1}^{n} t_{i}^{2} \sum_{i=1}^{n} t_{i}^{2} \cdot \omega_{i}}{n \sum_{i=1}^{n} t_{i}{ }^{4}-\left(\sum_{i=1}^{n} t_{i}^{2}\right)^{2}} ; \quad b=\frac{n \cdot \sum_{i=1}^{n} t_{i}^{2} \cdot \omega_{i}-\sum_{i=1}^{n} t_{i}^{2} \cdot \sum_{i=1}^{n} \omega_{i}}{\sum_{i=1}^{n} t_{i}^{2}} ; c=\frac{n \sum_{i=1}^{n} t_{i}^{4}-\left(\sum_{i=1}^{n} t_{i}^{2}\right)^{2}}{}
\end{gathered}
$$


Table 6 The "conglomerate" of the statistical data which joins under the incidence of the reflection concerning the quadratic pattern for the volum of Ferrari cars selled at worldwide level

\begin{tabular}{|c|c|c|c|c|c|c|c|}
\hline \multirow{2}{*}{ YEARS } & \multirow{2}{*}{$\begin{array}{c}\text { FERRARI } \\
\text { WORLDWIDE } \\
\text { NUMBER OF } \\
\text { VEHICLES } \\
\left(\omega_{i}\right)\end{array}$} & \multicolumn{6}{|c|}{ PARABOLIC TENDENCY } \\
\hline & & $t_{i}$ & $t_{i}^{2}$ & $t_{i}^{4}$ & $t_{i}^{2} \omega_{i}$ & $\omega_{t_{i}}=a+b t_{i}+c t_{i}^{2}$ & $\mid \omega_{i}-\omega_{t_{i}}$ \\
\hline 2006 & 5671 & -6 & 36 & 1296 & 204156 & 6107,461538 & 436 \\
\hline 2007 & 6465 & -5 & 25 & 625 & 161625 & 6172,340659 & 293 \\
\hline 2008 & 6587 & -4 & 16 & 256 & 105392 & 6267,987013 & 319 \\
\hline 2009 & 6250 & -3 & 9 & 81 & 56250 & 6394,400599 & 144 \\
\hline 2010 & 6461 & -2 & 4 & 16 & 25844 & 6551,581418 & 91 \\
\hline 2011 & 7001 & -1 & 1 & 1 & 7001 & 6739,50947 & 262 \\
\hline 2012 & 7318 & 0 & 0 & 0 & 0 & 6958,244755 & 360 \\
\hline 2013 & 6922 & 1 & 1 & 1 & 6922 & 7207,727272 & 286 \\
\hline 2014 & 7255 & 2 & 4 & 16 & 29020 & 7487,977023 & 233 \\
\hline 2015 & 7664 & 3 & 9 & 81 & 68976 & 7798,994006 & 135 \\
\hline 2016 & 8014 & 4 & 16 & 256 & 128224 & 8140,778221 & 127 \\
\hline 2017 & 8398 & 5 & 25 & 625 & 209950 & 8513,32967 & 115 \\
\hline 2018 & 9251 & 6 & 36 & 1296 & 333036 & 8916,648351 & 334 \\
\hline TOTAL & 93257 & & 182 & 4550 & 1336396 & 93257 & 3135 \\
\hline
\end{tabular}

$$
\begin{gathered}
a=\frac{\sum_{i=1}^{n} t_{i}^{4} \sum_{i=1}^{n} \xi_{i}-\sum_{i=1}^{n} t_{i}^{2} \sum_{i=1}^{n} t_{i}^{2} \cdot \xi_{i}}{n \sum_{i=1}^{n} t_{i}^{4}-\left(\sum_{i=1}^{n} t_{i}^{2}\right)^{2}}=\frac{4550 \cdot 93257-182 \cdot 1336396}{13 \cdot 4550-182^{2}}=6958,244755 \\
b=\frac{\sum_{i=1}^{n} \xi_{i} t_{i}}{\sum_{i=1}^{n} t_{i}^{2}}=\frac{42606}{182}=234,0989011 \\
c=\frac{n \cdot \sum_{i=1}^{n} t_{i}^{2} \cdot \xi_{i}-\sum_{i=1}^{n} t_{i}^{2} \cdot \sum_{i=1}^{n} \xi_{i}}{n \sum_{i=1}^{n} t_{i}{ }^{4}-\left(\sum_{i=1}^{n} t_{i}^{2}\right)^{2}}=\frac{13 \cdot 1336396-182 \cdot 93257}{13 \cdot 4550-182^{2}}=15,38361638 \\
v_{I I}=\left[\frac{\sum_{i=-m}^{m}\left|\omega_{i}-\omega_{t_{i}}^{I I}\right|}{n}: \frac{\sum_{i=-m}^{m} \omega_{i}}{n}\right] \cdot 100=\frac{\sum_{i=-m}^{m}\left|\omega_{i}-\omega_{t_{i}}^{I I}\right|}{\sum_{i=-m}^{m} \omega_{i}} \cdot 100=\frac{3135}{93257} \cdot 100=3,36 \%
\end{gathered}
$$

- if the "sophisticated design" for $\omega$ characteristic, namely the Ferrari worldwide sales, is this mirroring $\omega_{t_{i}}=a b^{t_{i}}, a$ and $b$ will be [3]:

$$
\left\{\begin{array}{l}
n \cdot \lg a+\lg b \cdot \sum_{i=1}^{n} t_{i}=\sum_{i=1}^{n} \lg \omega_{i} \\
\lg a \sum_{i=1}^{n} t_{i}+\lg b \cdot \sum_{i=1}^{n} t_{i}^{2}=\sum_{i=1}^{n} t_{i} \cdot \lg \omega_{i}
\end{array}\right.
$$




$$
\begin{aligned}
& \lg a=\frac{\left|\begin{array}{cc}
\sum_{i=1}^{n} \lg \omega_{i} & \sum_{i=1}^{n} t_{i} \\
\sum_{i=1}^{n} t_{i} \lg \omega_{i} & \sum_{i=1}^{n} t_{i}{ }^{2}
\end{array}\right|}{\left|\begin{array}{cc}
n & \sum_{i=1}^{n} t_{i} \\
\sum_{i=1}^{n} t_{i} & \sum_{i=1}^{n} t_{i}{ }^{2}
\end{array}\right|}=\frac{\sum_{i=1}^{n} \lg \omega_{i} \sum_{i=1}^{n} t_{i}{ }^{2}-\sum_{i=1}^{n} t_{i} \lg \omega_{i} \sum_{i=1}^{n} t_{i}}{n \sum_{i=1}^{n} t_{i}{ }^{2}-\left(\sum_{i=1}^{n} t_{i}\right)^{2}} \\
& \lg b=\frac{\left|\begin{array}{cc}
n & \sum_{i=1}^{n} \lg \omega_{i} \\
\sum_{i=1}^{n} t_{i} & \sum_{i=1}^{n} t_{i} \lg \omega_{i}
\end{array}\right|}{\left|\begin{array}{cc}
n & \sum_{i=1}^{n} t_{i} \\
\sum_{i=1}^{n} t_{i} & \sum_{i=1}^{n} t_{i}{ }^{2}
\end{array}\right|}=\frac{n \cdot \sum_{i=1}^{n} t_{i} \lg \omega_{i}-\sum_{i=1}^{n} \lg \omega_{i} \sum_{i=1}^{n} t_{i}}{n \sum_{i=1}^{n} t_{i}{ }^{2}-\left(\sum_{i=1}^{n} t_{i}\right)^{2}}
\end{aligned}
$$

Table 7 The „conglomerate” of the statistical data which joins under the incidence of the reflection

\begin{tabular}{|c|c|c|c|c|c|c|}
\hline \multirow{2}{*}{ YEARS } & \multirow{2}{*}{$\begin{array}{c}\text { FERRARI } \\
\text { WORLDWIDE } \\
\text { NUMBER OF } \\
\text { VEHICLES } \\
\left(\omega_{i}\right)\end{array}$} & \multicolumn{5}{|c|}{ EXPONENTIAL TENDENCY } \\
\hline & & $\lg \omega_{i}$ & $t_{i} \lg \omega_{i}$ & $\begin{array}{c}\lg \omega_{t_{i}}= \\
=\lg a+t_{i} \lg b\end{array}$ & $\omega_{t_{i}}=a b^{t_{i}}$ & $\omega_{i}-\omega_{t_{i}}$ \\
\hline 2006 & 5671 & 3,753659647 & $-22,52195788$ & 3,768256187 & 5864,840249 & 194 \\
\hline 2007 & 6465 & 3,810568529 & $-19,05284265$ & 3,782241521 & 6056,776122 & 408 \\
\hline 2008 & 6587 & 3,818687663 & $-15,27475065$ & 3,796226855 & 6254,993389 & 332 \\
\hline 2009 & 6250 & 3,795880017 & $-11,38764005$ & 3,810212189 & 6459,697619 & 210 \\
\hline 2010 & 6461 & 3,810299741 & $-7,620599482$ & 3,824197523 & 6671,101109 & 210 \\
\hline 2011 & 7001 & 3,845160078 & $-3,845160078$ & 3,838182857 & 6889,423102 & 112 \\
\hline 2012 & 7318 & 3,864392405 & 0 & 3,852168191 & 7114,890016 & 203 \\
\hline 2013 & 6922 & 3,840231595 & 3,840231595 & 3,866153525 & 7347,735679 & 426 \\
\hline 2014 & 7255 & 3,860637417 & 7,621274834 & 3,880138859 & 7588,201574 & 333 \\
\hline 2015 & 7664 & 3,884455960 & 11,65336649 & 3,894124193 & 7836,537082 & 173 \\
\hline 2016 & 8014 & 3,903849338 & 15,61539735 & 3,908109527 & 8092,999750 & 79 \\
\hline 2017 & 8398 & 3,924175870 & 19,62087935 & 3,922094861 & 8357,855552 & 40 \\
\hline 2018 & 9251 & 3,966188681 & 23,79713209 & 3,936080195 & 8631,379165 & 620 \\
\hline TOTAL & 93257 & 50,078186480 & 2,545330915 & & & 3340 \\
\hline
\end{tabular}
concerning the exponential pattern for the volum of Ferrari cars selled at worldwide level

$$
\begin{gathered}
\lg a=\frac{50,07818648 \cdot 182-2,545330915 \cdot 0}{13 \cdot 182-0^{2}}=3,85216191 \\
\lg b=\frac{13 \cdot 2,545330915-50,07818648 \cdot 0}{13 \cdot 182-0^{2}}=0,013985334 \\
v_{\exp }=\left[\frac{\sum_{i=-m}^{m}\left|\omega_{i}-\omega_{t_{i}}^{\exp }\right|}{n}: \frac{\sum_{i=-m}^{m} \omega_{i}}{n}\right] \cdot 100=\frac{\sum_{i=-m}^{m}\left|\omega_{i}-\omega_{t_{i}}^{\exp }\right|}{\sum_{i=-m}^{m} \omega_{i}} \cdot 100=\frac{3340}{93257} \cdot 100=3,58 \% \\
v_{I I}=3,36 \%<v_{\exp }=3,58 \%<v_{I}=3,74 \%
\end{gathered}
$$


The "design of the rally" concerning the projection for $\omega$ factor, which belongs to the Ferrari worldwide sales, is a quadratic pattern $\omega_{t_{i}}=a+b \cdot t_{i}+c t_{i}^{2}$

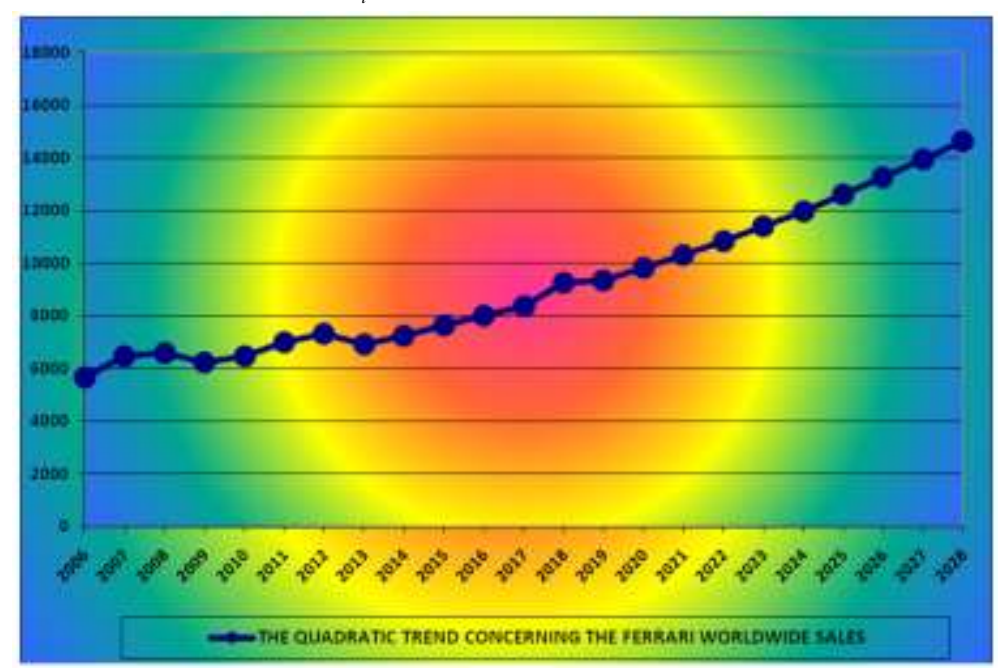

Graph 2 The design of the quadratic pattern regarding the worldwide sales for the Ferrari cars

Because the design of the modelling for the values of Lamborghini and Ferrari cars selled at worldwide level shows a quadratic pattern $\xi_{t_{i}}=a+b \cdot t_{i}+c t_{i}^{2}$, the forecasts projected will be:

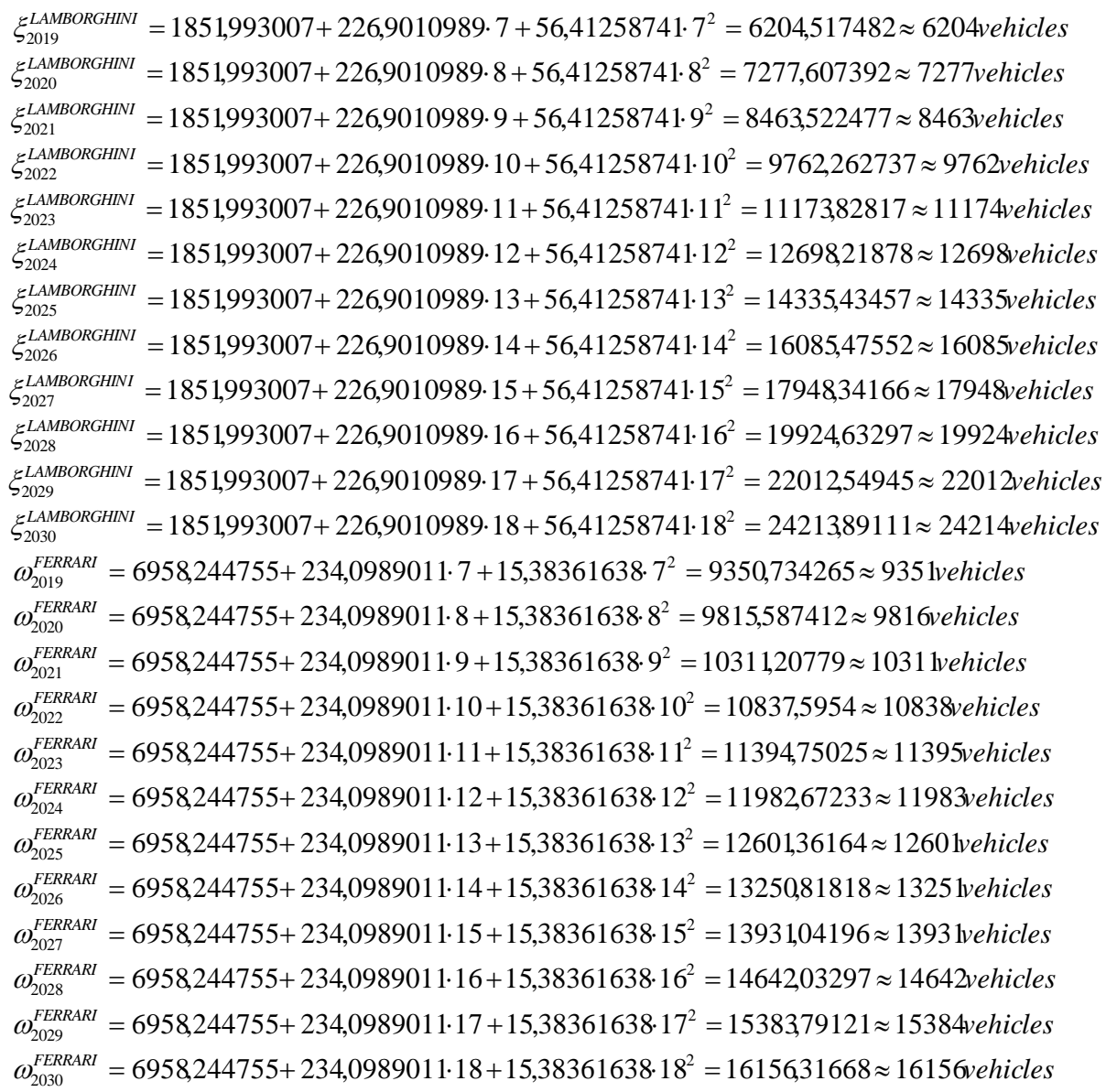


Table 8 Statistical pol position leadership between Lamborghini \& Ferrari, in the period 2006-2030

\begin{tabular}{|c|c|c|c|c|c|c|c|c|}
\hline & \multirow{3}{*}{$\begin{array}{l}\text { LAMBORGHINI } \\
\text { WORLDWIDE } \\
\text { NUMBER } \\
\text { OF VEHICLES }\end{array}$} & \multirow{3}{*}{$\begin{array}{c}\text { FERRARI } \\
\text { WORLDWIDE } \\
\text { NUMBER } \\
\text { OF VEHICLES }\end{array}$} & \multicolumn{6}{|c|}{ THE DYNAMICS CONCERNING THE LEADERSHIP } \\
\hline & & & \multirow[t]{2}{*}{$I_{\text {Ferrari / Lamb }}^{\text {CARS }}$} & \multirow[t]{2}{*}{$I_{\text {Lambo / Ferrar }}^{C A R S}$} & \multirow{2}{*}{\multicolumn{2}{|c|}{$\begin{array}{l}\text { (RELATIVE RISES) } \\
\text { (\%) }\end{array}$}} & $\Delta_{\text {Ferrari / Lamb }}^{\text {CARS }}$ & $\Delta_{\text {Lambo/Ferrari }}^{C A R S}$ \\
\hline & & & & & & & \multicolumn{2}{|c|}{$\begin{array}{c}\text { (ABSOLUTE RISES) } \\
\text { (NUMBER OF VEHICLES) }\end{array}$} \\
\hline 2006 & 2087 & 5671 & 271,73 & & 171,73 & & 3584 & \\
\hline 2007 & 2406 & 6465 & 268,70 & & 168,70 & & 4059 & \\
\hline 2008 & 2430 & 6587 & 271,07 & & 171,07 & & 4157 & \\
\hline 2009 & 1515 & 6250 & 412,54 & & 312,54 & & 4735 & \\
\hline 2010 & 1302 & 6461 & 496,24 & & 396,24 & & 5159 & \\
\hline 2011 & 1602 & 7001 & 437,02 & & 337,02 & & 5399 & \\
\hline 2012 & 2083 & 7318 & 352,32 & & 252,32 & & 5235 & \\
\hline 2013 & 2121 & 6922 & 326,36 & & 226,36 & & 4801 & \\
\hline 2014 & 2530 & 7255 & 286,76 & & 186,76 & & 4725 & \\
\hline 2015 & 3245 & 7664 & 236,18 & & 136,18 & & 4419 & \\
\hline 2016 & 3457 & 8014 & 231,82 & & 131,82 & & 4557 & \\
\hline 2017 & 3815 & 8398 & 220,13 & & 120,13 & & 4583 & \\
\hline 2018 & 5750 & 9251 & 160,89 & & 60,89 & & 3501 & \\
\hline 2019 & 6244 & 9351 & 149,76 & & 49,76 & & 3107 & \\
\hline 2020 & 7277 & 9816 & 134,89 & & 34,89 & & 2539 & \\
\hline 2021 & 8463 & 10311 & 121,84 & & 21,84 & & 1848 & \\
\hline 2022 & 9762 & 10838 & 111,02 & & 11,02 & & 1076 & \\
\hline 2023 & 11174 & 11395 & 101,98 & & 1,98 & & 221 & \\
\hline 2024 & 12698 & 11983 & & 105,97 & & 5,97 & & 715 \\
\hline 2025 & 14335 & 12601 & & 113,76 & & 13,76 & & 1734 \\
\hline 2026 & 16085 & 13251 & & 121,39 & & 21,39 & & 2834 \\
\hline 2027 & 17948 & 13931 & & 128,83 & & 28,83 & & 4017 \\
\hline 2028 & 19924 & 14642 & & 136,07 & & 36,07 & & 5282 \\
\hline 2029 & 22012 & 15384 & & 143,08 & & 43,08 & & 6628 \\
\hline 2030 & 24214 & 16156 & & 149,88 & & 49,88 & & 8058 \\
\hline
\end{tabular}

\section{Conclusions}

The profile of the pattern which paints the values of the worldwide sales regarding the Lamborghini cars, between 2006-2018, unveils a propensity to the quadratic representation. The configuration of the design which is projected on the volum of the worldwide sales concerning the Ferrari cars, between 20062018 , reveals a proclivity to the quadratic tendency. We can see that in 2018, Ferrari had a rise with 60,89\%, comparative to Lamborghini, of the value concerning the number of cars selled in the worldwide sphere. Also, in 2024 Lamborghini will obtain a rise with 5,97 \% comparative to Ferrari, of the value regarding the number of cars selled at the worldwide plan. If we analysis the forecasts regarding the number of cars selled at the worlwide level, in 2030 Lamborghini will achieve a growth with 49,88\% comparative to Ferrari. So, we can say that, there is a strong meeting in pol position concerning the leadership between these two great "hypersonic" leaders and this competition will be forever with effects on the adrenalin for the lovers of super exotic cars.

\section{References}

[1]. Bruce P., Bruce A. -"Practical Statistics For Data Scientists: 50 Essential Concepts", O'Reilly Media Inc Publishing House, New York, 2017.

[2]. Crocetta C. - "Theoretical and Applied Statistics", Springer Publishing House, Berlin, 2019.

[3]. Gauss C.F. - „Disquisitiones Arithmeticae and other papers on number theory”, english translation Springer Publishing House, New York, 1986.

[4]. Lamm J. - „Exotic Cars, First Gear”, MotorBooks Publishing House, New York, 2008.

[5]. Mann J., Codling S. - „Form Follows Function: The Art of the Supercar”, MotorBooks Publishing House, New York, 2011.

[6]. Perna C., Pratezi M., Ruiz Gazen A. - „Studies in Theoretical and Applied Statistics”, Springer Publishing House, Berlin, 2016.

[7]. Pfanzagl J. - „Mathematical Statistics”, Springer Publishing House, Berlin, 2017.

[8]. Philip S. -„Top Gear: Dream Cars, The Hot 100”, BBC Books Publishing House, London, 2016. 